\title{
New perspectives of analog and digital simulations of fractional order systems
}

\author{
ABDELFATAH CHAREF, MOHAMED CHAREF, ABDELBAKI DJOUAMBI and ALINA VODA
}

\begin{abstract}
In the recent decades, fractional order systems have been found to be useful in many areas of physics and engineering. Hence, their efficient and accurate analog and digital simulations and numerical calculations have become very important especially in the fields of fractional control, fractional signal processing and fractional system identification. In this article, new analog and digital simulations and numerical calculations perspectives of fractional systems are considered. The main feature of this work is the introduction of an adjustable fractional order structure of the fractional integrator to facilitate and improve the simulations of the fractional order systems as well as the numerical resolution of the linear fractional order differential equations. First, the basic ideas of the proposed adjustable fractional order structure of the fractional integrator are presented. Then, the analog and digital simulations techniques of the fractional order systems and the numerical resolution of the linear fractional order differential equation are exposed. Illustrative examples of each step of this work are presented to show the effectiveness and the efficiency of the proposed fractional order systems analog and digital simulations and implementations techniques.
\end{abstract}

Key words: adjustable fractional operators, Charef approximation, fractional differential equation, fractional integrator, fractional systems

\section{Introduction}

The subject of fractional order systems has gained considerable importance in the recent decades due mainly to their numerous applications in various fields of applied science and engineering [10], [23], [24], [33]. Nowadays well known concepts in the fields of control system, signal processing and identification are being extended for the development of their fractional order counterparts as emerging topics [2], [12], [30], [31], [34]. Hence, the fractional order systems efficient, reliable and accurate simulations and numerical calculations have become very important research topics. The considerable

A. Charef (corresponding author; e-mail: afcharef@yahoo.com) and M. Charef are with Laboratoire de Traitement du Signal Département d'Electronique, Université des Frères Mentouri - Constantine, Route Ain El-bey, Constantine 25011, Algeria. A. Djouambi is with Département d'Electronique, Université Larbi Ben M'Hidi, Oum-El-Bouaghi 04000, Algeria. A. Voda is with Université Grenoble Alpes, CNRS, GIPSA-lab, F-38000 Grenoble, France.

Received 19.01.2017. 
attention given to such systems is to establish a fractional system theory so it may be accessible to the general science and engineering communities.

A major problem with fractional systems is their time domain simulations which are more difficult compared to the regular systems because they are basically infinite dimensional systems due to their fractional orders. This is challenging and researchers working in this domain have developed different interesting methods to simulate them. There are broadly two major approaches for the time domain simulations of the fractional order systems: analytical and numerical methods. The purpose of the analytical method is to obtain an explicit expression for the general responses of the fractional order systems. Yet, analytical responses are often not simple to obtain. Only some closed form analog or digital approximation expressions of the responses of the fractional systems have been developed [3], [4], [5], [14], [16], [20], [25], [27], [28]. On the contrary, the goal of the numerical method is the development of a robust and stable numerical scheme for the responses of the fractional order systems. A great deal of effort has been expended in this research axis leading to a variety of techniques. But, there is no proposed efficient numerical method which simultaneously achieves speed, accuracy, and ease of simulation. Two types of numerical approaches have been developed for the simulation of the fractional order systems. The first method is based on the digital approximation of the irrational analog transfer function of the fractional order system leading to a recurrent equation. The digital approximation can be obtained by indirect or direct discretization. In indirect discretization technique two steps are required, first analog frequency domain fitting of the irrational analog transfer function is made then one of the $s$ to $z$ transform methods such as Euler, Tustin or Al-Alaoui is used for the discretization. The direct discretization method is based on the application of power series in the $z$ domain of the Euler operator, Tustin operator or Al-Alaoui operator to the fractional differentiation of the irrational analog transfer function of the fractional system [1], [9], [15], [17], [18], [24], [29], [36]. The most used direct discretization is the Grunwald-Letnikov definition of the fractional differentiation [24]. Fractional differential equations have also been the focus of many mathematicians. Consequently, considerable attention has been given to their numerical solutions [11], [19], [21], [22], [32], [35]. However, these methods may not be interesting from an engineering approach at least in terms of simulation and implementation of fractional systems.

In this article, new simulations and numerical calculations perspectives of the fractional order systems based on an adjustable fractional order structure of the fractional integrator are considered. First, using Charef's approximation method [6], we will derive an adjustable fractional order rational function approximation of the analog fractional integrator $s^{-m}$ ( $m$ is any real positive number) where the poles of the rational function are calculated only one time for $m=0.5$, which means that they are completely independent of the parameter $m$ [7]. Analog and digital simulation structures made up of two parts will be derived to simulate the fractional order integrator $s^{-m}$ for any real positive number $m$. The right part is a fixed structure designed only once for $m=0.5$ and it will be used for any fractional order $m>0$. The left part is a structure composed of an ensemble of functions depending on the fractional order $m$ only. Then, the proposed simulation 
structure of the fractional integrator will be used to derive analog and digital simulations of the fractional order systems represented by linear fractional differential equations. At last, the numerical solution of the linear fractional order differential equations is obtained. Illustrative examples of this work are presented to show the effectiveness and the efficiency of the proposed analog and digital simulations techniques and resolution of the fractional order systems.

\section{Fractional order integrator: adjustable fractional order structure}

The analog fractional order integrator is represented by the following irrational transfer function:

$$
G_{I}(s)=\frac{1}{s^{m}}, \text { for } m>0 .
$$

In a given frequency band of interest $\left[\omega_{L}, \omega_{H}\right]$ and a given integer number $N$, the rational function approximation of the fractional order operator $G_{I}(s)$ can be expressed by the following equation [6], [8], [13]:

$$
G_{I}(s)=\frac{1}{s^{m}} \cong \frac{1}{\left(\omega_{c}\right)^{m}} \frac{\prod_{i=1}^{N-1}\left[1+\frac{s}{z_{i}(m)}\right]}{\prod_{i=1}^{N}\left[1+\frac{s}{p_{i}(m)}\right]}
$$

the poles $p_{i}(m)$ (for $\left.i=1,2, \ldots, N\right)$ and the zeros $z_{i}(m)$ (for $i=1,2, \ldots,(N-1)$ ) of the above approximation are given as:

$$
p_{i}(m)=\omega_{c} 10^{\left(\frac{2 i-1-m}{m(1-m)}\right)} \varepsilon, \quad z_{i}(m)=\omega_{c} 10^{\left(\frac{2 i-1+m}{m(1-m)}\right) \varepsilon}
$$

where

$$
\varepsilon=\frac{m(1-m)}{2\left(N+\frac{1-m}{2}\right)}\left[\log _{10}\left(\omega_{\max } / \omega_{c}\right)\right]
$$

is the approximation error and the frequencies $\omega_{c}$ and $\omega_{\max }$ such that $\omega_{c}=\gamma \omega_{L}$ (for $10^{-5} \leqslant \gamma \leqslant 1$ ) and $\omega_{\max }=\theta \omega_{H}$ (for $1 \leqslant \theta \leqslant 10^{5}$ ).

The rational function of equation (2) can be decomposed as:

$$
G_{I}(s)=\frac{1}{s^{m}} \cong \sum_{i=1}^{N} \frac{h_{i}(m)}{\left(1+\frac{s}{p_{i}(m)}\right)}
$$

where the residues $h_{i}(m)$ (for $\left.i=1,2, \ldots, N\right)$ are calculated as:

$$
\left.h_{i}(m)=\frac{1}{\left(\omega_{c}\right)^{m}} \frac{\prod_{\substack { j=1 \\
\begin{subarray}{c}{j=1 \\
j \neq i{ j = 1 \\
\begin{subarray} { c } { j = 1 \\
j \neq i } }\end{subarray}}^{N-1}\left(1-\frac{p_{i}(m)}{z_{j}(m)}\right)}{p_{j}(m)}\right) .
$$


The adjustable fractional order structure of the rational function approximation of the fractional integrator is realized such that all the poles $p_{i}$ (for $i=1,2, \ldots, N$ ) are completely independent of the fractional order m. It has been shown in [7] that the best value of the parameter $m$ used to calculate the poles $p_{i}(m)$ is $m=0.5$. So, equation (4) is rewritten as:

$$
G_{I}(s)=\frac{1}{s^{m}} \cong \sum_{i=1}^{N} \frac{h_{i}(m)}{\left(1+\frac{s}{p_{i}}\right)}
$$

where the poles $p_{i}=p_{i}(m=0.5)$ (for $\left.i=1,2, \ldots, N\right)$ are given as:

$$
p_{i}=\omega_{c} 10^{(8 i-6) \varepsilon} \text {. }
$$

In the rational function approximation of equation (2), the error and the frequency $\omega_{c}$ normally depend on the fractional order $m$. But, $\varepsilon$ ) and $\omega_{c}$ are calculated for $m=0.5$ which means that $\varepsilon=\varepsilon(0.5)$ and $\omega_{c}=\omega_{c}(0.5)$. For $m \neq 0.5, \varepsilon(m)$ and $\omega_{c}(m)$ have to be adjusted to guarantee that $p_{1}(0.5)=p_{1}(m)$. From [6], $\varepsilon(m)=4 m(1-m) \varepsilon$ and $\omega_{c}(m)=\omega_{c} 10^{[4 m-2] \varepsilon}$. So, the fixed poles $p_{i}$ (for $\left.i=1,2, \ldots, N\right)$ and the zeros $z_{i}(m)$ (for $i=1,2, \ldots,(N-1))$ of equation (3) are given as:

$$
p_{i}=\omega_{c} 10^{(8 i-6) \varepsilon}, \quad z_{i}(m)=\omega_{c} 10^{(8 i-4+4 m) \varepsilon} .
$$

Then, using the expressions of the poles and the zeros of equation (8) the residues $h_{i}(m)$ (for $i=1,2, \ldots, N$ ) of equation (5) are derived as:

$$
h_{i}(m)=\frac{1}{\left[\omega_{c} 10^{(4 m-2) \varepsilon}\right]^{m}} \frac{\prod_{\substack { j=1 \\
\begin{subarray}{c}{j=1^{N} \\
j \neq i{ j = 1 \\
\begin{subarray} { c } { j = 1 ^ { N } \\
j \neq i } }\end{subarray}}^{N-1}\left(1-10^{8(i-j) \varepsilon}\right)}{\left.\prod^{8(i-j-m) \varepsilon}\right)}
$$

\subsection{Adjustable fractional order structure recap}

In a given frequency band of interest $\left[\omega_{L}, \omega_{H}\right]$ and a given integer number $N$, we have:

$$
G_{I}(s)=\frac{1}{s^{m}} \cong \sum_{i=1}^{N} \frac{h_{i}(m)}{\left(1+\frac{s}{p_{i}}\right)}, \text { for } m>0
$$

the fixed poles $p_{i}$ and the residues $h_{i}(m)$ (for $i=1,2, \ldots, N$ ) of the approximation are given as:

$$
p_{i}=\omega_{c} 10^{(8 i-6) \varepsilon}, \quad h_{i}(m)=\frac{1}{\left[\omega_{c} 10^{(4 m-2) \varepsilon}\right]^{m}} \frac{\prod_{\substack { j=1 \\
\begin{subarray}{c}{j=1^{N} \\
j \neq i{ j = 1 \\
\begin{subarray} { c } { j = 1 ^ { N } \\
j \neq i } }\end{subarray}}^{N-1}\left(1-10^{8(i-j-m) \varepsilon}\right)}{\left.\prod^{8(i-j) \varepsilon}\right)}
$$

where $\varepsilon=\frac{1}{(8 N+2)}\left[\log _{10}\left(\frac{\omega_{\max }}{\omega_{c}}\right)\right], \omega_{c}=\gamma \omega_{L},\left(10^{-5} \leqslant \gamma \leqslant 1\right)$ and $\omega_{\max }=\theta \omega_{H},\left(1 \leqslant \theta \leqslant 10^{5}\right)$. 


\subsection{Analog simulation}

The rational function approximation of the fractional integrator $s^{-m}$ of equation (10) is:

$$
\frac{Y(s)}{E(s)}=G_{I}(s)=\frac{1}{s^{m}} \cong \sum_{i=1}^{N} \frac{h_{i}(m)}{\left(1+\frac{s}{p_{i}}\right)}
$$

The output of the fractional integrator is then given by:

$$
Y(s)=\sum_{i=1}^{N} \frac{h_{i}(m)}{1+\frac{s}{p_{i}}} E(s)=\sum_{i=1}^{N}\left(h_{i}(m) E(s)\right) \frac{1}{1+\frac{s}{p_{i}}}=\sum_{i=1}^{N} V_{i}(s)
$$

with

$$
V_{i}(s)=\left(h_{i}(m) E(s)\right)\left(\frac{1}{1+\frac{s}{p_{i}}}\right), \text { for } i=1,2, \ldots, N .
$$

In the time domain, for $i=1,2, \ldots, N, v_{i}(t)$ is simulated by the first order differential equation

$$
\frac{d v_{i}(t)}{d t}=-p_{i} v_{i}(t)+p_{i}\left(h_{i}(m) e(t)\right) \text {. }
$$

Then the analog simulation of the fractional order integrator $s^{-m}$ is given as:

$$
\left\{\begin{array}{l}
y(t)=\sum_{i=1}^{N} v_{i}(t) \\
\frac{d v_{i}(t)}{d t}=-p_{i} v_{i}(t)+\left(p_{i} h_{i}(m)\right) e(t)
\end{array}\right\}, \text { for } m>0 \text { and } i=1,2, \ldots, N
$$

Fig. 1 shows the analog simulation of the fractional integrator $s^{-m}$ using equation (14). The right part of the simulation structure representing the second expression of equation (14) is a fixed structure made of first order sub-systems which are completely independent of the fractional order $m$. So, it can be used for the simulation of the fractional integrator of any fractional order $m>0$. The left part is a structure composed of an ensemble of functions depending on the fractional order m only.

\subsection{Digital simulation}

The analog simulation of the fractional integrator $s^{-m}$ is given in equation (14). So, the digital simulation of the fractional integrator can be obtained from equation (14) as follows:

$$
y(k)=\sum_{i=1}^{N} v_{i}(k)
$$

and from the second expression of equation (14), we have (for $i=1,2, \ldots, N$ ):

$$
V_{i}(s)=\frac{1}{1+\frac{s}{p_{i}}}\left(h_{i}(m)\right) E(s) \text {. }
$$




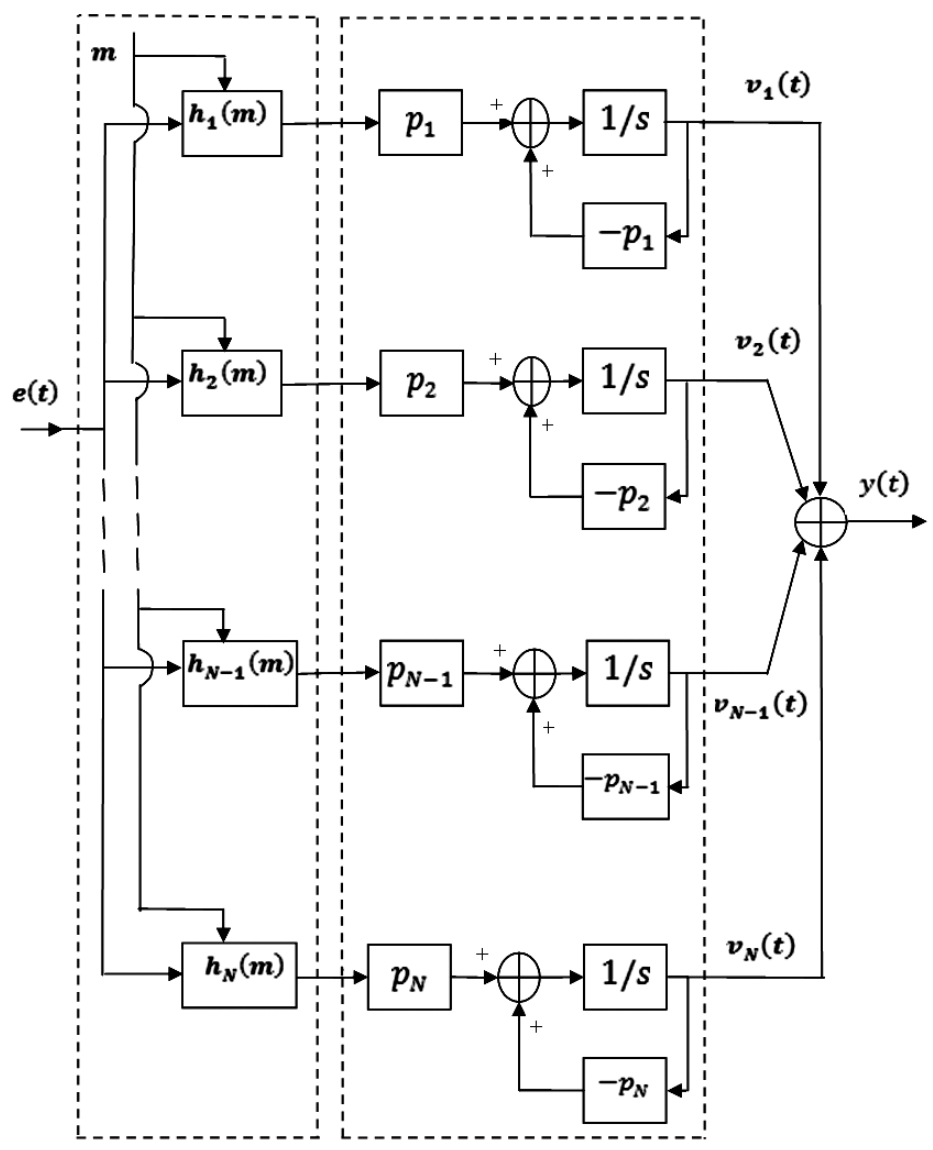

Figure 1: Proposed analog simulation of the fractional integrator $s^{-m}(m>0)$

The $Z$ transform of the analog transfer function

$$
\frac{1}{1+\frac{s}{p_{i}}}, \text { for } i=1,2, \ldots, N
$$

of equation (16) with zero order hold ( $\mathrm{ZOH})$ is obtained as follows [26]:

$$
Z\left\{(\mathrm{ZOH})\left(\frac{1}{1+\frac{s}{p_{i}}}\right)\right\}=\left(1-z^{-1}\right) Z\left\{\frac{1}{s\left(1+\frac{s}{p_{i}}\right)}\right\}=\frac{\left(1-\delta_{i}\right) z^{-1}}{1-\delta_{i} z^{-1}}
$$

where $\delta_{i}=\exp \left(-T p_{i}\right)$ (for $\left.i=1,2, \ldots, N\right)$ with $T$ the sampling period. So, from equations (16) and (18) we can write that (for $i=1,2, \ldots, N$ ):

$$
V_{i}(z)=\left(\frac{\left(1-\delta_{i}\right) z^{-1}}{1-\delta_{i} z^{-1}}\right)\left(h_{i}(m)\right) E(z) .
$$


Rearranging equation (19), we will get (for $i=1,2, \ldots, N$ ):

$$
V_{i}(z)=\delta_{i} z^{-1} V_{i}(z)+\left(1-\delta_{i}\right)\left(h_{i}(m)\right)\left(z^{-1} E(z)\right) .
$$

In the time domain, $v_{i}(k)$ (for $i=1,2, \ldots, N$ ) is given as:

$$
v_{i}(k)=\delta_{i} v_{i}(k-1)+\left(h_{i}(m)\right)\left(1-\delta_{i}\right) e(k-1)
$$

Then, the digital simulation of the fractional integrator $s^{-m}$ (for $m>0$ ) is given as follows:

$$
\left\{\begin{aligned}
y(k) & =\sum_{i=1}^{N} v_{i}(k) \\
v_{i}(k) & =\delta_{i} v_{i}(k-1)+\left(h_{i}(m)\right)\left(1-\delta_{i}\right) e(k-1), \text { for } i=1,2, \ldots, N
\end{aligned}\right.
$$

Fig. 2 shows the digital simulation of the fractional integrator $s^{-m}$ (for $m>0$ ). Because it is derived from the analog one the digital simulation structure is also made of two parts. The right part representing the second expression of equation (22) is a fixed structure which can be used for the digital simulation of the fractional integrator of any fractional order $m>0$. The left part is a structure composed of the same ensemble of functions of the analog structure.

\subsection{Illustrative example}

To show the effectiveness and the usefulness of the proposed method, we will consider the rational function approximation of the analog fractional order integrators $s^{-0.63}$ and $s^{-1.74}$ in the frequency band $\left[\omega_{L}, \omega_{H}\right]=[0.001 \mathrm{rad} / \mathrm{s}, 1000 \mathrm{rad} / \mathrm{s}]$ for $N=20$. From equation (10), we get:

$$
\begin{gathered}
\frac{1}{\mathrm{~s}^{0.63}} \cong \sum_{\mathrm{i}=1}^{20} \frac{\mathrm{h}_{\mathrm{i}}(0.63)}{1+\frac{s}{p_{i}}} \\
\frac{1}{s^{1.74}} \cong \sum_{i=1}^{20} \frac{h_{i}(1.74)}{1+\frac{s}{p_{i}}} .
\end{gathered}
$$

For $\omega_{c}=0.001, \omega_{L}=10^{-6}$ and $\omega_{\max }=1000$ and $\omega_{H}=10^{6}$ we have $\varepsilon=0.0741$. Then, from equation (11), the poles and the residues (for $i=1,2, \ldots, 20$ ) are: 


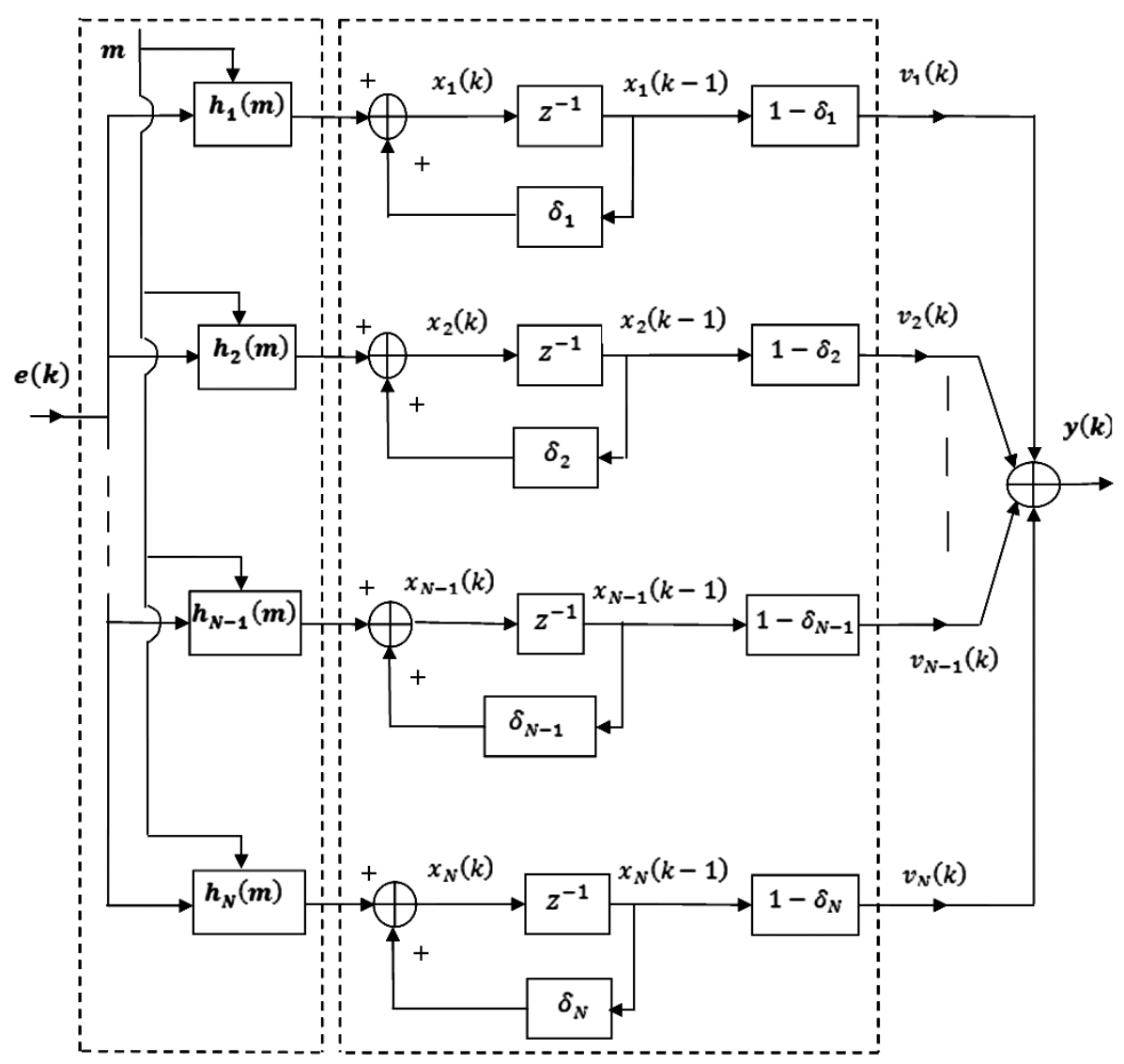

Figure 2: Proposed digital simulation of the fractional integrator $s^{-m}(m>0)$

$$
\begin{gathered}
p_{i}=10^{(0.5928 i-6.4446)} \\
h_{i}(0.63)=(1.7550 e-4)\left[\frac{\prod_{j=1}^{19}\left(1-10^{0.5928(i-j-0.63)}\right)}{\prod_{\substack{j=1 \\
j \neq i}}^{20}\left(1-10^{0.5928(i-j)}\right)}\right] \\
h_{i}(1.74)=(1.5823 e-10)\left[\frac{\prod_{j=1}^{19}\left(1-10^{0.5928(i-j-1.74)}\right)}{\prod_{\substack{j=1 \\
j \neq i}}^{20}\left(1-10^{0.5928(i-j)}\right)}\right] .
\end{gathered}
$$


We note that for both fractional order integrators $s^{-0.63}$ and $s^{-1.74}$, the poles of their rational function approximations are the same only the residues are different. In this example, we emphasize that for any fractional order integrator $s^{-m}(m>0)$ the poles of its rational function approximation will be the ones of equation (25). Figs. 3 and 4 show the Bode plots of the ideal analog fractional order integrators $s^{-0.63}$ and $s^{-1.74}$ and of their rational function approximations of equations (23) and (24).
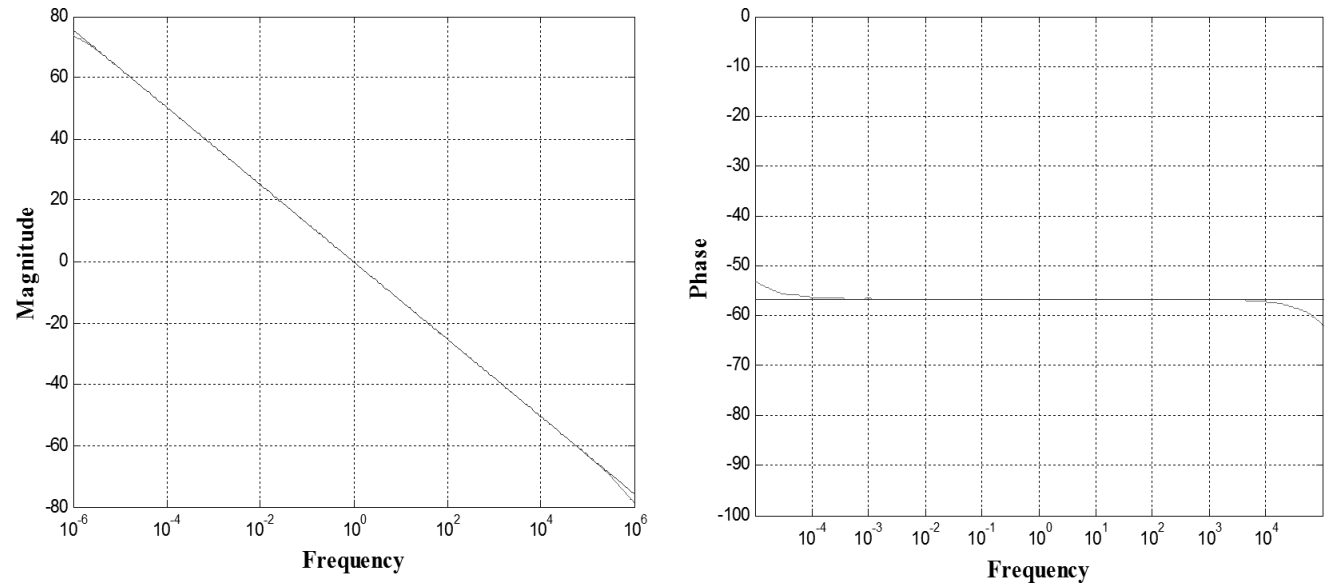

Figure 3: Bode plot of the fractional integrator $s^{-0.63}$ and its rational function approximation
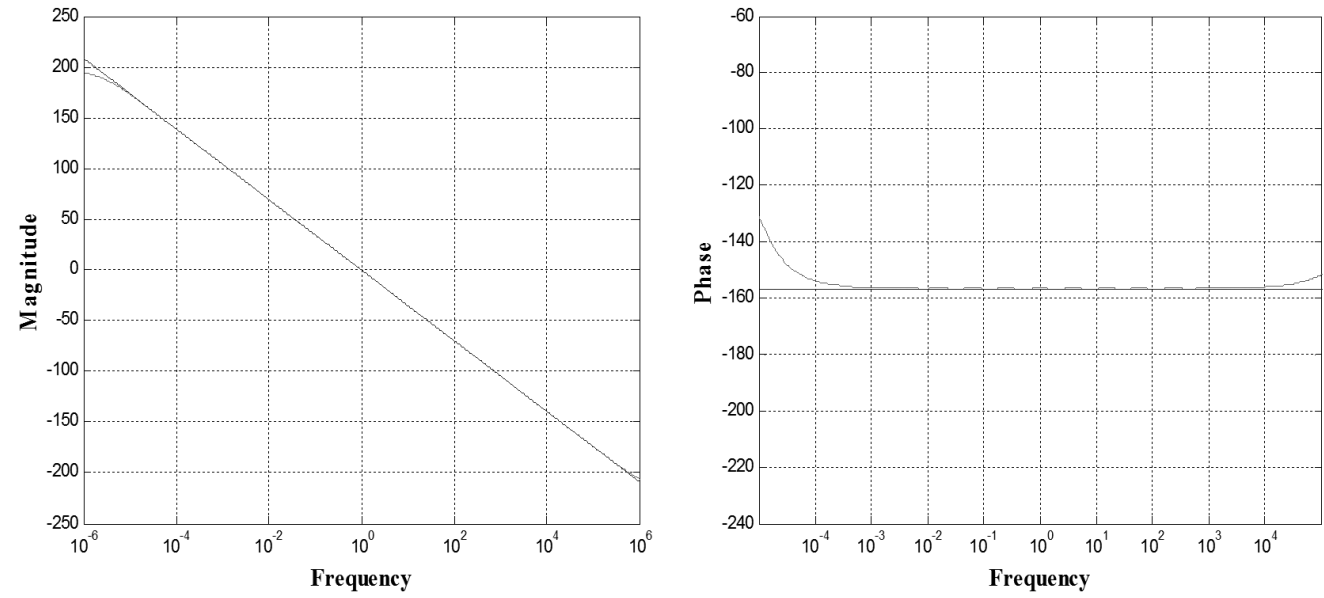

Figure 4: Bode plot of the fractional integrator $s^{-1.74}$ and its rational function approximation 


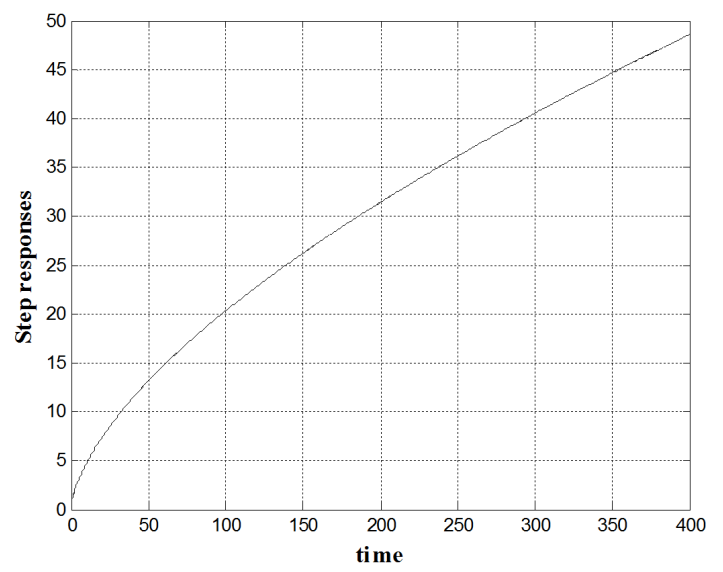

Figure 5: Step responses of the fractional order integrators $s^{-0.63}$ using MATLAB function fode_sol () and the proposed approximation structure

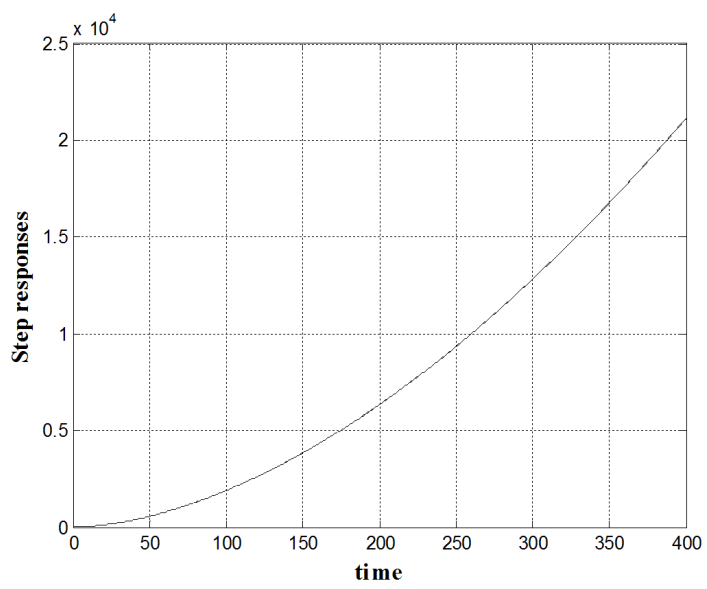

Figure 6: Step responses of the fractional order integrators $s^{-1.74}$ using MATLAB function fode_sol () and the proposed approximation structure

From Figs 3 and 4, we can easily see that the Bode plots of the ideal ana$\log$ fractional order integrators $s^{-0.63}$ and $s^{-1.74}$ and their corresponding rational function approximations are quite overlapping in the frequency band of interest $\left[\omega_{L}, \omega_{H}\right]=[0.001 \mathrm{rad} / \mathrm{s}, 1000 \mathrm{rad} / \mathrm{s}]$. Figs 5 and 6 show the step responses of the fractional integrators $s^{-0.63}$ and $s^{-1.74}$ using the MATLAB function fode_sol() based on the Grunwald-Letnikov's fractional differentiation definition for the numerical solution of the linear fractional differential equation [24] and using the proposed approximation structure. The equations used for the step responses are given as: 
Fractional order integrator $s^{-0.63}$

- using MATLAB function fode_sol ( ) [24]:

solve the fractional differential equation $\frac{d^{0.63} y(t)}{d t^{0.63}}=u(t)$ to get the step response of the fractional integrator $s^{-0.63}$ as:

$\mathrm{a}=[1] ; \mathrm{na}=[0.63] ; \mathrm{b}=[1] ; \mathrm{nb}=[0] ; \mathrm{t}=0: 0.1: 4001$;

$\mathrm{u}=\operatorname{ones}(\operatorname{size}(\mathrm{t}))$,

$\mathrm{y}=$ fode_sol $(\mathrm{a}, \mathrm{na}, \mathrm{b}, \mathrm{nb}, \mathrm{u}, \mathrm{t})$

- using the proposed approximation structure:

For $i=1,2, \ldots, 20$, solve the 20 regular first order differential equations:

$$
\left\{\begin{aligned}
\frac{d v_{i}(t)}{d t} & =-10^{(0.5928 i-6.4446)} v_{i}(t)+ \\
& +10^{(0.5928 i-10.2003)} \frac{\prod_{\substack{j=1 \\
19}}^{20}\left(1-10^{0.5928(i-j-0.63)}\right)}{\prod_{\substack{j=1 \\
j \neq i}}^{20}\left(1-10^{0.5928(i-j)}\right)} e(t) \\
y(t) & =\sum_{i=1}^{20} v_{i}(t)
\end{aligned}\right.
$$

Fractional order integrator $s^{-1.74}$

- using MATLAB function fode_sol ( ) [24]:

solve the fractional differential equation $\frac{d^{1.74} y(t)}{d t^{1.74}}=u(t)$ to get the step response of the fractional integrator $s^{-1.74}$ as:

$\mathrm{a}=[1] ; \mathrm{na}=[1.74] ; \mathrm{b}=[1] ; \mathrm{nb}=[0] ; \mathrm{t}=0: 0.1: 4001 ;$

$\mathrm{u}=\operatorname{ones}(\operatorname{size}(\mathrm{t}))$,

$\mathrm{y}=$ fode_sol $\left._{-} \mathrm{a}, \mathrm{na}, \mathrm{b}, \mathrm{nb}, \mathrm{u}, \mathrm{t}\right)$

- using the proposed approximation structure:

For $i=1,2, \ldots, 20$, solve the 20 regular first order differential equations:

$$
\left\{\begin{aligned}
\frac{d v_{i}(t)}{d t} & =-10^{(0.5928 i-6.4446)} v_{i}(t)+ \\
& +10^{(0.5928 i-16.2447)} \frac{\prod_{\substack{19 \\
1=1}}^{20}\left(1-10^{0.5928(i-j-1.74)}\right)}{\prod_{\substack{j=1 \\
j \neq i}}^{20}\left(1-10^{0.5928(i-j)}\right)} e(t) \\
y(t) & =\sum_{i=1}^{20} v_{i}(t)
\end{aligned}\right.
$$


From Figs. 5 and 6, we can easily see that the step responses of fractional integrators $s^{-0.63}$ and $s^{-1.74}$ using the proposed simulation structure and the MATLAB function fode_sol ( ) are exactly the same.

\section{Linear fractional order system: New structure}

A linear single input single output (SISO) fractional order system is described by the following linear fractional order differential equation [24]:

$$
\sum_{i=0}^{L} a_{i} D^{\alpha_{i}} y(t)=\sum_{j=0}^{M} b_{j} D^{\beta_{j}} e(t)
$$

where $e(t)$ is the input, $y(t)$ is the output, the derivative orders $\alpha_{i}(0 \leqslant i \leqslant L)$ and $\beta_{i}$ $(0 \leqslant j \leqslant M-1)$ are constant real positive numbers such that $\alpha_{L-1}<\cdots<\alpha_{1}<\alpha_{0}$, $\beta_{M-1}<\cdots<\beta_{1}<\beta_{0}, \beta_{0} \leqslant \alpha_{0}$, and $\alpha_{L}=\beta_{M}=0$; the model parameters $a_{i}(1 \leqslant i \leqslant L)$ and $b_{i}(0 \leqslant j \leqslant M)$ are constant real numbers with $a_{0}=1$. With zero initial conditions, the fractional system transfer function is given as [24]:

$$
G(s)=\frac{Y(s)}{E(s)}=\frac{\sum_{j=0}^{M} b_{j} s^{\beta_{j}}}{\sum_{i=0}^{L} a_{i} s^{\alpha_{i}}} .
$$

From equation (29), we can write:

$$
G(s)=\frac{Y(s)}{E(s)}=\frac{\sum_{j=0}^{M} b_{j} s^{\beta_{j}}}{s^{\alpha_{0}}+\sum_{i=1}^{L} a_{i} s^{\alpha_{i}}} .
$$

So, we will have:

$$
\begin{gathered}
{\left[s^{\alpha_{0}}+\sum_{i=1}^{L} a_{i} s^{\alpha_{i}}\right] Y(s)=\left[\sum_{j=0}^{M} b_{j} s^{\beta_{j}}\right] E(s)} \\
s^{\alpha_{0}} Y(s)=-\left[\sum_{i=1}^{L} a_{i} s^{\alpha_{i}}\right] Y(s)+\left[\sum_{j=0}^{M} b_{j} s^{\beta_{j}}\right] E(s) .
\end{gathered}
$$

$Y(s)$ can then be obtained as:

$$
Y(s)=-\left[\sum_{i=1}^{L} a_{i} \frac{1}{s^{\alpha_{0}-\alpha_{i}}}\right] Y(s)+\left[\sum_{j=0}^{M} b_{j} \frac{1}{s^{\alpha_{0}-\beta_{j}}}\right] E(s) .
$$


From section 2.1, in a given frequency band of interest $\left[\omega_{L}, \omega_{H}\right]$ and a given integer number $N$, the fractional order integrators $\frac{1}{s^{\alpha_{0}-\alpha_{i}}}$ and $\frac{1}{s^{\alpha_{0}-\beta_{j}}}$, for $i=1,2, \ldots, L$ and for $j=0,2, \ldots, M$, are approximated as:

$$
\begin{gathered}
\frac{1}{s^{\alpha_{0}-\alpha_{i}}} \cong \sum_{q=1}^{N} \frac{h_{q}\left(\alpha_{0}-\alpha_{i}\right)}{\left(1+s / p_{q}\right)} \\
\frac{1}{s^{\alpha_{0}-\beta_{j}}} \cong \sum_{q=1}^{N} \frac{h_{q}\left(\alpha_{0}-\beta_{j}\right)}{\left(1+s / p_{q}\right)}
\end{gathered}
$$

The poles $p_{q}$ and the residues $h_{q}(\sigma)$ (for $q=1,2, \ldots, N$ and $\sigma=\left(\alpha_{0}-\alpha_{i}\right)$ or $\sigma=$ $\left.\left(\alpha_{0}-\beta_{\mathrm{j}}\right)\right)$ are given as:

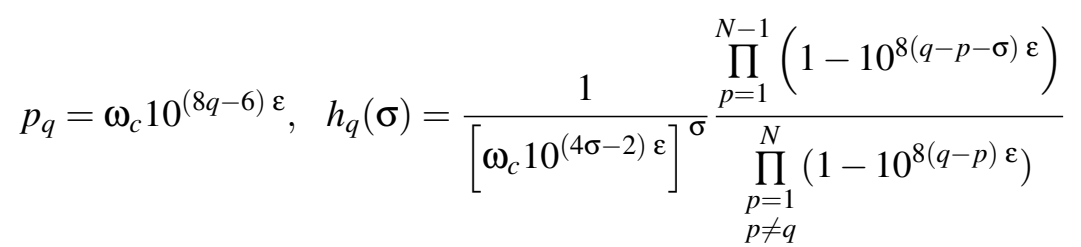

where $\varepsilon=\frac{1}{(8 N+2)}\left[\log _{10}\left(\omega_{\max } / \omega_{c}\right)\right], \omega_{c}=\gamma \omega_{L},\left(10^{-5} \leqslant \gamma \leqslant 1\right)$ and $\omega_{\max }=\theta \omega_{H},(1 \leqslant$ $\left.\theta \leqslant 10^{5}\right)$.

Then equation (33) can be rewritten as:

$$
\begin{gathered}
Y(s)=-\left[\sum_{i=1}^{L} a_{i} \sum_{q=1}^{N} \frac{h_{q}\left(\alpha_{0}-\alpha_{i}\right)}{1+\frac{s}{p_{q}}}\right] Y(s)+\left[\sum_{j=0}^{M} b_{j} \sum_{q=1}^{N} \frac{h_{q}\left(\alpha_{0}-\beta_{j}\right)}{1+\frac{s}{p_{q}}}\right] E(s) \\
Y(s)=-\sum_{q=1}^{N}\left(\sum_{\mathrm{i}=1}^{\mathrm{L}} a_{i} \mathrm{~h}_{\mathrm{q}}\left(\alpha_{0}-\alpha_{\mathrm{i}}\right)\right)\left(\frac{Y(s)}{1+\frac{s}{p_{q}}}\right)+\sum_{q=1}^{N}\left(\sum_{\mathrm{j}=0}^{\mathrm{M}} b_{j} \mathrm{~h}_{\mathrm{q}}\left(\alpha_{0}-\beta_{\mathrm{j}}\right)\right)\left(\frac{E(s)}{1+\frac{s}{p_{q}}}\right) \\
Y(s)=\sum_{q=1}^{N}\left(A_{q} Y(s)\right)\left(\frac{1}{1+\frac{s}{p_{q}}}\right)+\sum_{q=1}^{N}\left(B_{q} E(s)\right)\left(\frac{1}{1+\frac{s}{p_{q}}}\right)
\end{gathered}
$$

where the coefficients $A_{q}$ and $B_{q}$, for $q=1,2, \ldots, N$, are given by the following expressions:

$$
\begin{aligned}
& A_{q}=-\sum_{i=1}^{L} a_{i} h_{q}\left(\alpha_{0}-\alpha_{i}\right) \\
& B_{q}=\sum_{j=0}^{M} b_{j} h_{q}\left(\alpha_{0}-\beta_{j}\right) .
\end{aligned}
$$




\subsection{Analog simulation}

A linear SISO fractional order system is described by the linear fractional order differential equation of equation (28) as:

$$
\sum_{i=0}^{L} a_{i} D^{\alpha_{i}} y(t)=\sum_{j=0}^{M} b_{j} D^{\beta_{j}} e(t) .
$$

From equation (39), its solution is given by the following expression:

$$
Y(s)=\sum_{q=1}^{N}\left[A_{q} Y(s)+B_{q} E(s)\right]\left(\frac{1}{1+\frac{s}{p_{q}}}\right)=\sum_{q=1}^{N} V_{q}(s)
$$

where the variables $V_{q}(s)$, for $q=1,2, \ldots, N$, are defined as follows:

$$
V_{q}(s)=\left(A_{q} Y(s)+B_{q} E(s)\right)\left(\frac{1}{1+\frac{s}{p_{q}}}\right) .
$$

So, in the time domain, each variable $v_{q}(t)$ (for $q=1,2, \ldots, N$ ) is the solution of the following first order differential equation:

$$
\frac{d v_{q}(t)}{d t}=-p_{q} v_{q}(t)+p_{q}\left(A_{q} y(t)+B_{q} e(t)\right) .
$$

Hence, from equations (42) and (44), the analog simulation of the linear SISO fractional order system described by the linear fractional order differential equation of equation (41) is given by:

$$
\left\{\begin{array}{l}
y(t)=\sum_{q=1}^{N} v_{q}(t) \\
\frac{d v_{q}(t)}{d t}=-p_{q} v_{q}(t)+p_{q}\left(A_{q} y(t)+B_{q} e(t)\right), \text { for } q=1,2, \ldots, N
\end{array}\right.
$$

Fig. 7 shows the proposed analog simulation using equation (45) of the linear SISO fractional order system described by the linear fractional order differential equation of equation (41). The right part of Fig. 7 of the proposed simulation structure representing the second expression of equation (45) is a fixed structure made of first order sub-systems which are completely independent of the fractional orders $\alpha_{i}(0 \leqslant i \leqslant L)$ and $\beta_{i}(0 \leqslant j \leqslant$ $M)$ of the linear fractional order differential equation of equation (41). Then, this right part of the proposed analog simulation can be used for the simulation of any linear SISO fractional order system described by the linear fractional order differential equation of equation (41). The left part of Fig. 7 is an ensemble of functions depending only on the derivative orders $\alpha_{i}(0 \leqslant i \leqslant L)$ and $\beta_{i}(0 \leqslant j \leqslant M)$ and the model coefficients $a_{i}$ (for $0 \leqslant i \leqslant L)$ and $b_{i}(0 \leqslant j \leqslant M)$ of equation (41). 


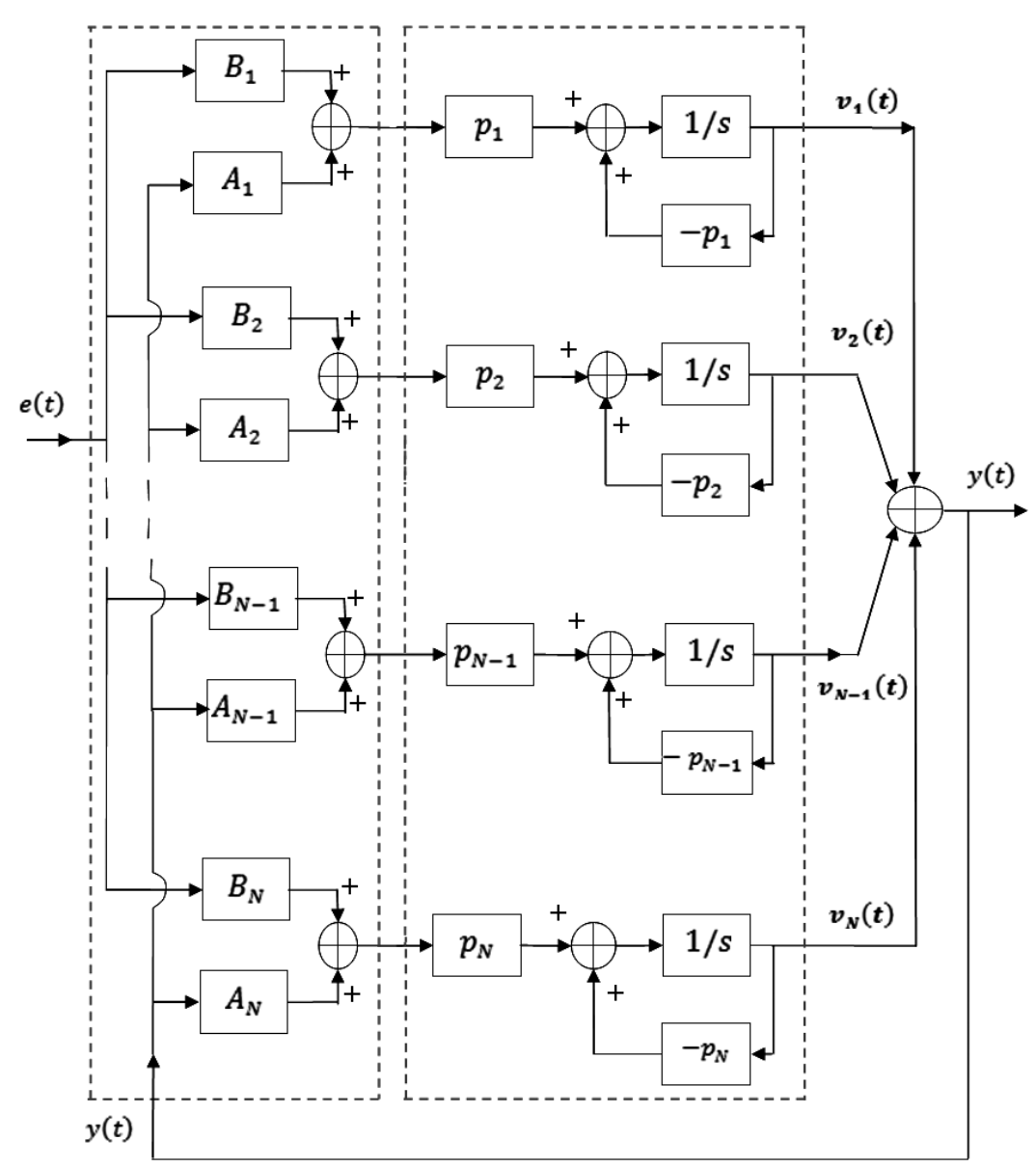

Figure 7: Analog simulation structure of the linear SISO fractional order system

\subsection{Numerical resolution of the linear fractional differential equation}

A linear SISO fractional system is described by the linear fractional differential equation of equation (28) as:

$$
\sum_{i=0}^{L} a_{i} D^{\alpha_{i}} y(t)=\sum_{j=0}^{M} b_{j} D^{\beta_{j}} e(t) .
$$

The analog solution of the above differential equation is given by equation (42) as:

$$
Y(s)=\sum_{q=1}^{N} V_{q}(s)
$$


where the variables $V_{q}(s)$, for $q=1,2, \ldots, N$, are given from equation (43) by the expression:

$$
V_{q}(s)=\left[A_{q} Y(s)+B_{q} E(s)\right] \frac{1}{1+\frac{s}{p_{q}}} .
$$

The $Z$ transform of the analog transfer function $\frac{1}{1+\frac{s}{p_{q}}}$ (for $\left.q=1,2, \ldots, N\right)$ of equation (48) with zero order hold ( $\mathrm{ZOH})$ is then obtained as follows [26]:

$$
Z\left\{(\mathrm{ZOH})\left(\frac{1}{1+\frac{s}{p_{q}}}\right)\right\}=\left(1-z^{-1}\right) Z\left\{\frac{1}{s\left(1+\frac{s}{p_{q}}\right)}\right\}=\frac{\left(1-\delta_{q}\right) z^{-1}}{1-\delta_{q} z^{-1}}
$$

where $\delta_{q}=\exp \left(-T p_{q}\right)$ (for $\left.i=q, 2, \ldots, N\right)$ and $T$ is the sampling period. So, from equation (48) we can write that (for $q=1,2, \ldots, N$ ):

$$
V_{q}(z)=\left(A_{q} Y(z)+B_{q} E(z)\right)\left(\frac{\left(1-\delta_{q}\right) z^{-1}}{1-\delta_{q} z^{-1}}\right) .
$$

Rearranging equation (50), we will get (for $q=1,2, \ldots, N$ ):

$$
V_{q}(z)=\delta_{q} z^{-1} V_{q}(z)+\left(1-\delta_{q}\right)\left(A_{q} z^{-1} Y(z)+B_{q} z^{-1} E(z)\right) .
$$

In the time domain, $v_{q}(k)$ (for $q=1,2, \ldots, N$ ) is given as:

$$
V_{q}(z)=\delta_{q} z^{-1} V_{q}(z)+\left(1-\delta_{q}\right)\left(A_{q} z^{-1} Y(z)+B_{q} z^{-1} E(z)\right) .
$$

So, the numerical solution of the linear fractional order differential equation of equation (46) is obtained from equations (47) and (52) as follows:

$$
\left\{\begin{array}{l}
v_{q}(k)=\delta_{q} v_{q}(k-1)+\left(1-\delta_{q}\right)\left(A_{q} y(k-1)+B_{q} e(k-1)\right), \text { for } q=1,2, \ldots, N \\
y(k)=\sum_{q=1}^{N} v_{q}(k)
\end{array}\right.
$$

where, for $q=1,2, \ldots, N, A_{q}=-\sum_{i=1}^{L} a_{i} h_{q}\left(\alpha_{0}-\alpha_{i}\right), B_{q}=\sum_{j=0}^{M} b_{j} h_{q}\left(\alpha_{0}-\beta_{j}\right)$ and $\delta_{q}=$ $\exp \left(-T p_{q}\right)$. The parameters $p_{q}$ and $h_{q}(\sigma)$ (for $\left.q=1,2, \ldots, N\right)$ and $\sigma=\left(\alpha_{0}-\alpha_{\mathrm{i}}\right)$ or $\sigma=$ $\left(\alpha_{0}-\beta_{j}\right)$ (for $i=1,2, \ldots, L$ and for $j=0,2, \ldots, M$ ) are obtained for a given frequency band of interest $\left[\omega_{L}, \omega_{H}\right]$ and a given integer number $N$ as follows:

$$
p_{q}=\omega_{c} 10^{(8 q-6) \varepsilon}, \quad h_{q}(\sigma)=\frac{1}{\left[\omega_{c} 10^{(4 \sigma-2) \varepsilon}\right]} \frac{\prod_{p=1}^{N-1}\left(1-10^{8(q-p-\sigma) \varepsilon}\right)}{\prod_{\substack{p=1 \\ p \neq q}}^{N}\left(1-10^{8(q-p) \varepsilon}\right)}
$$

where $\varepsilon=\frac{1}{(8 N+2)}\left[\log _{10}\left(\frac{\omega_{\max }}{\omega_{c}}\right)\right], \omega_{c}=\gamma \omega_{L}\left(10^{-5} \leqslant \gamma \leqslant 1\right)$ and $\omega_{\max }=\theta \omega_{H}(1 \leqslant \theta \leqslant$ $\left.10^{5}\right)$. 


\subsection{Digital simulation}

A linear SISO fractional order system is described by the linear fractional order differential equation of equation (46) as:

$$
\sum_{i=0}^{L} a_{i} D^{\alpha_{i}} y(t)=\sum_{j=0}^{M} b_{j} D^{\beta_{j}} e(t)
$$

The numerical solution of the above differential equation can be obtained from equation (47) as:

$$
Y(z)=\sum_{q=1}^{N} V_{q}(z)
$$

where the variables $V_{q}(z)$, for $q=1,2, \ldots, N$, are given from equation (50) by the expressions:

$$
V_{q}(z)=\left(A_{q} Y(z)+B_{q} E(z)\right)\left(\frac{\left(1-\delta_{q}\right) z^{-1}}{1-\delta_{q} z^{-1}}\right) .
$$

Rearranging equation (57), we will get (for $i=1,2, \ldots, N$ ):

$$
\frac{V_{q}(z)}{A_{q} Y(z)+B_{q} E(z)}=\frac{\left(1-\delta_{q}\right) z^{-1}}{1-\delta_{q} z^{-1}} .
$$

The variables $X_{q}(z)$, for $q=1,2, \ldots, N$, are such that the above equation can be rewritten as :

$$
\frac{V_{q}(z)}{X_{q}(z)} \cdot \frac{X_{q}(z)}{\left(A_{q} Y(z)+B_{q} E(z)\right)}=\frac{\left(1-\delta_{q}\right) z^{-1}}{1-\delta_{q} z^{-1}}
$$

Let $\frac{V_{q}(z)}{X_{q}(z)}=\left(1-\delta_{q}\right) z^{-1}$ and $\frac{X_{q}(z)}{\left(A_{q} Y(z)+B_{q} E(z)\right)}=\frac{1}{1-\delta_{q} z^{-1}}$, we will then have:

$$
V_{q}(z)=\left(1-\delta_{q}\right) z^{-1} X_{q}(z), \quad\left(1-\delta_{q} z^{-1}\right) X_{q}(z)=\left(A_{q} Y(z)+B_{q} E(z)\right) .
$$

So, in the time domain, (for $i=1,2, \ldots, N$ ), we will get:

$$
v_{q}(k)=\left(1-\delta_{q}\right) x_{q}(k-1), \quad x_{q}(k)=\delta_{q} x_{q}(k-1)+\left(A_{q} y(k)+B_{q} e(q)\right) .
$$

Hence, from equations (56) and (60), the digital simulation of the linear SISO fractional order system described by the linear fractional order differential equation of equation (55) is given by:

$$
\left\{\begin{array}{l}
y(k)=\sum_{q=1}^{N} v_{q}(k) \\
\left\{\begin{array}{l}
v_{q}(k)=\left(1-\delta_{q}\right) x_{q}(k-1) \\
x_{q}(k)=\delta_{q} x_{q}(k-1)+\left(A_{q} y(k)+B_{q} e(k)\right)
\end{array} \text { for } q=1,2, \ldots, N\right.
\end{array}\right.
$$


Fig. 8 shows the proposed digital simulation using equation (62) of the linear SISO fractional order system described by the differential equation of equation (55). This digital simulation structure is also made of two parts as the analog one. The right part, a structure of parallel first order sub-systems representing the two last expressions of equation (62), is completely independent of the fractional orders $\alpha_{i}(0 \leqslant i \leqslant L)$ and $\beta_{i}(0 \leqslant j \leqslant M)$ of the linear fractional order differential equation of equation (55). Because it is fixed, this part can be used for the digital simulation of any linear SISO fractional system described by the linear fractional order differential equation of equation (55). The left part of Fig. 8 is the same structure of the analog simulation of Fig. 7.

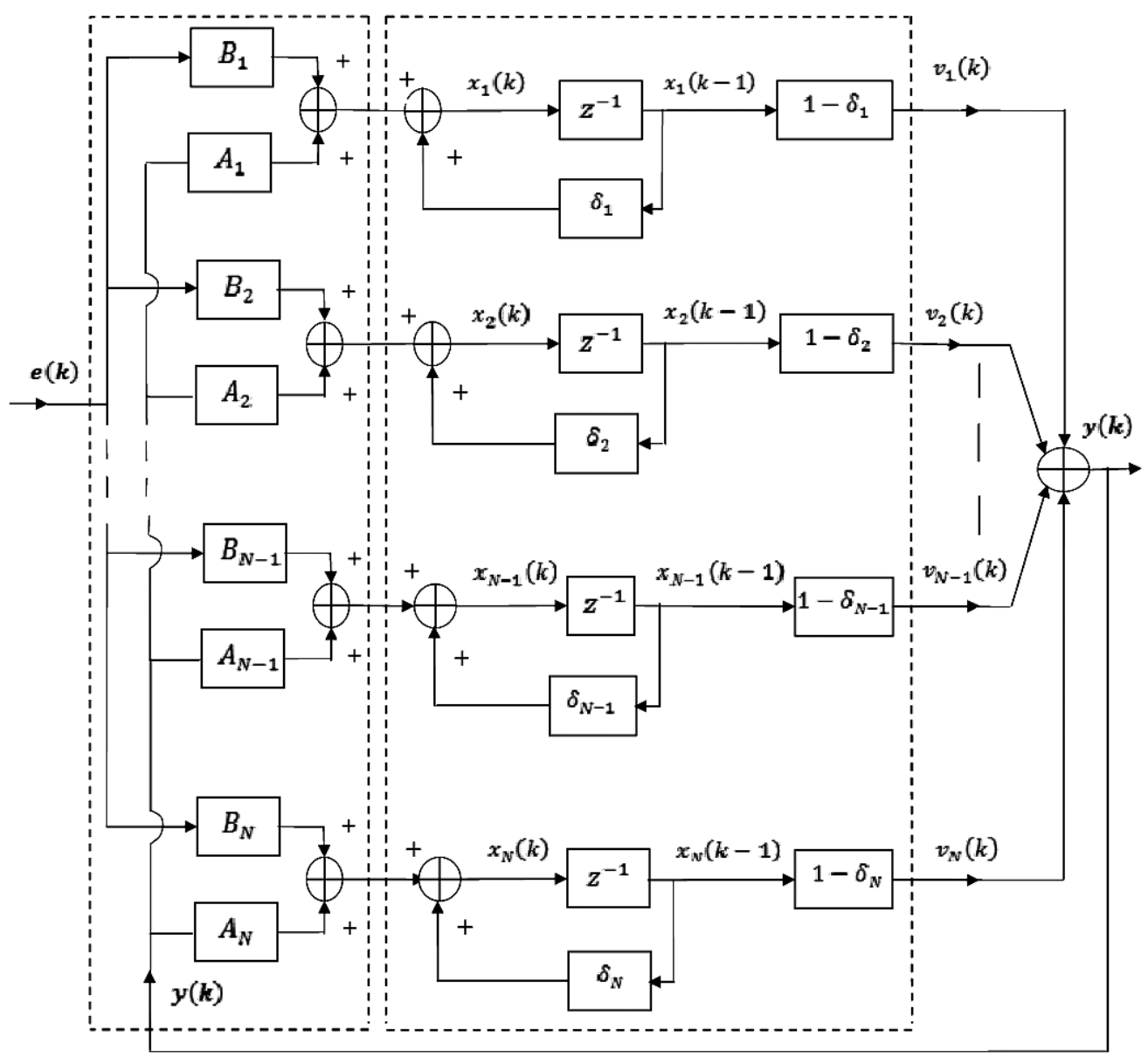

Figure 8: Digital simulation structure of the linear SISO fractional order system 


\subsection{Illustrative examples}

In the first example we will consider a linear SISO fractional order system described by the following linear fractional order differential equation:

$$
D^{\sqrt{5}} y(t)+20 D^{\sqrt{3}} y(t)+3 D^{0.85} y(t)+2 y(t)=3 D^{1.36} e(t)+8 e(t) .
$$

Using Laplace transform of the above linear fractional order differential equation with zero initial conditions, we will get:

$$
\begin{gathered}
\left(s^{\sqrt{5}}+20 s^{\sqrt{3}}+3 s^{0.85}+2\right) Y(s)=\left(3 s^{1.36}+8\right) E(s) \\
Y(s)=-\left(20 \frac{1}{s^{(\sqrt{5}-\sqrt{3})}}+3 \frac{1}{s^{(\sqrt{5}-0.85)}}+2 \frac{1}{s^{(\sqrt{5})}}\right) Y(s) \\
+\left(3 \frac{1}{s^{(\sqrt{5}-1.36)}}+8 \frac{1}{s^{(\sqrt{5})}}\right) E(s) .
\end{gathered}
$$

In the frequency band $\left[\omega_{\mathrm{L}}, \omega_{\mathrm{H}}\right]=[0.001 \mathrm{rad} / \mathrm{s}, 10000 \mathrm{rad} / \mathrm{s}]$ and for $N=22$. The rational function approximation of the fractional integrators $\frac{1}{s^{(\sqrt{5}-\sqrt{3})}}, \frac{1}{s^{(\sqrt{5}-0.85)}}, \frac{1}{s^{\sqrt{5}}}$ and $\frac{1}{s^{(\sqrt{5}-1.36)}}$ are given as:

$$
\begin{aligned}
\frac{1}{s^{(\sqrt{5}-\sqrt{3})} \cong \sum_{q=1}^{22} \frac{h_{q}(\sqrt{5}-\sqrt{3})}{1+\frac{s}{p_{q}}},} \quad \frac{1}{s^{(\sqrt{5}-0.85)} \cong \sum_{q=1}^{22} \frac{h_{q}(\sqrt{5}-0.85)}{1+\frac{s}{p_{q}}}} \\
\frac{1}{s^{(\sqrt{5})}} \cong \sum_{q=1}^{22} \frac{h_{q}(\sqrt{5})}{1+\frac{s}{p_{q}}}, \quad \frac{1}{s^{(\sqrt{5}-1.36)}} \cong \sum_{q=1}^{22} \frac{h_{q}(\sqrt{5}-1.36)}{1+\frac{s}{p_{q}}}
\end{aligned}
$$

We note that all the poles of the rational function approximation of the above four analog fractional order integrators are the same.

For $\omega_{c}=0.0005 \omega_{L}=5.0 \cdot 10^{-7}$ and $\omega_{\max }=100 \omega_{H}=10^{6}, \varepsilon=0.0691$, the poles $p_{q}$ and residues $h_{q}(\sqrt{5}-\sqrt{3}), h_{q}(\sqrt{5}-0.85), h_{q}(\sqrt{5})$ and $h_{q}(\sqrt{5}-1.36)$ (for $1 \leqslant$ $q \leqslant 22)$ are given as:

$$
\begin{gathered}
p_{q}=10^{(0.5529 q-6.7156)} \\
h_{q}(\sqrt{5}-\sqrt{3})=\left(1.4972 \cdot 10^{4}\right) \frac{\prod_{p=1}^{21}\left(1-10^{0.5529(q-p-\sqrt{5}+\sqrt{3})}\right)}{\prod_{\substack{p=1 \\
p \neq q}}^{22}\left(1-10^{0.5529(q-p)}\right)}
\end{gathered}
$$




$$
\begin{aligned}
& h_{q}(\sqrt{5}-0.85)=\left(2.4783 \cdot 10^{8}\right) \frac{\prod_{p=1}^{21}\left(1-10^{0.5529(q-p-\sqrt{5}+0.85)}\right)}{\prod_{\substack{p=1 \\
p \neq q}}^{22}\left(1-10^{0.5529(q-p)}\right)} \\
& h_{q}(\sqrt{5})=\left(1.0386 \cdot 10^{13}\right) \frac{\prod_{p=1}^{21}\left(1-10^{0.5529(q-p-\sqrt{5})}\right)}{\prod_{\substack{p=1 \\
p \neq q}}^{22}\left(1-10^{0.5529(q-p)}\right)} \\
& h_{q}(\sqrt{5}-1.36)=\left(2.6857 \cdot 10^{5}\right) \frac{\prod_{p=1}^{21}\left(1-10^{0.5529(q-p-\sqrt{5}+1.36)}\right)}{\prod_{\substack{p=1 \\
p \neq q}}^{22}\left(1-10^{0.5529(q-p)}\right)}
\end{aligned}
$$

Hence, the analog simulation of the linear SISO fractional order system described by the linear fractional order differential equation of equation (63) is given by:

$$
\left\{\begin{array}{l}
y(t)=\sum_{q=1}^{N} v_{q}(t) \\
\frac{d v_{q}(t)}{d t}=-p_{q} v_{q}(t)+p_{q}\left(A_{q} y(t)+B_{q} e(t)\right), \text { for } \mathrm{q}=1,2, \ldots, 22
\end{array}\right.
$$

where, for $1 \leqslant q \leqslant 22, p_{q}$ is as above; $A_{q}$ and $B_{q}$ are given as:

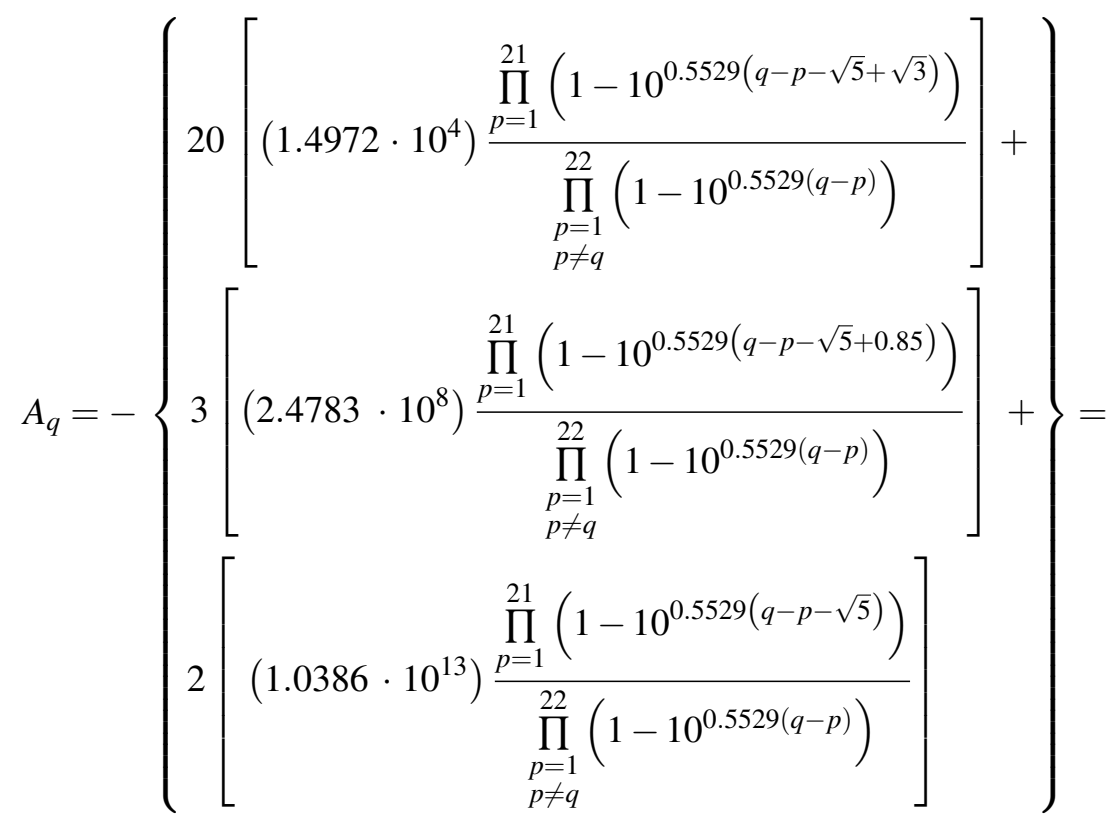




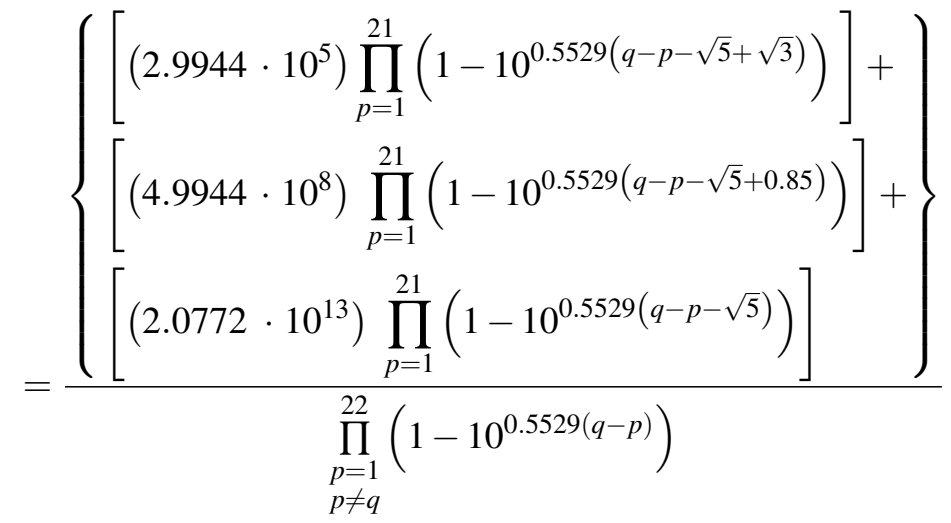

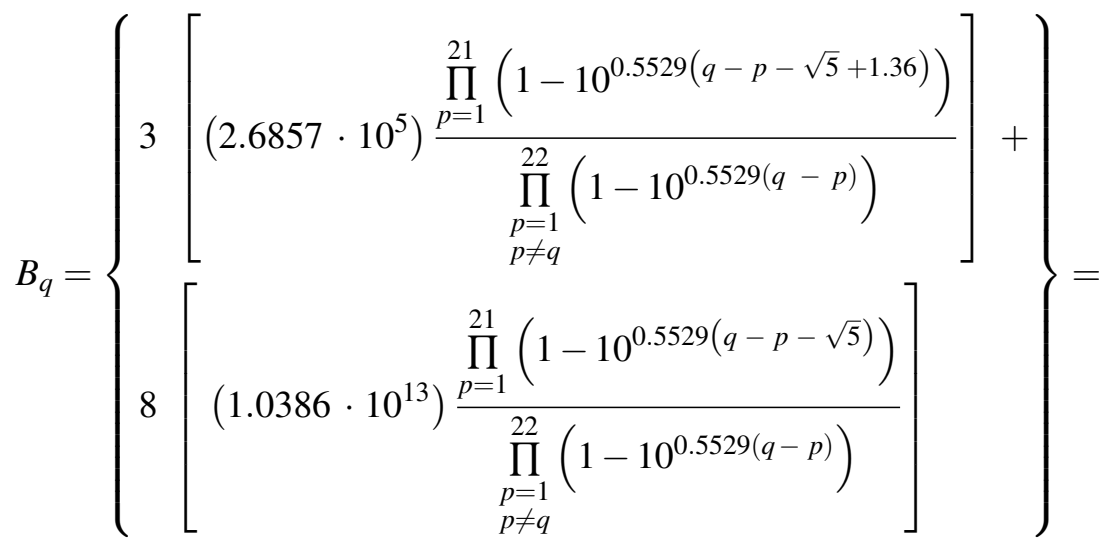

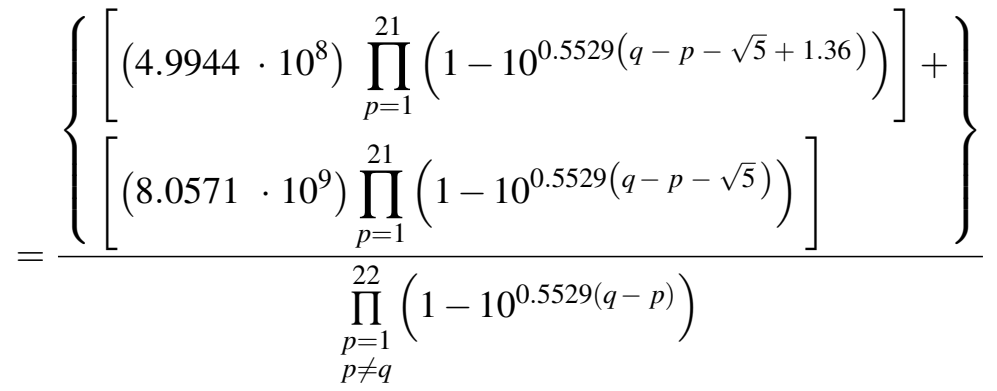

The numerical solution of the differential equation of equation (63) is given as follows:

$$
\left\{\begin{array}{l}
y(k)=\sum_{q=1}^{N} v_{q}(k) \\
v_{q}(k)=-\delta_{q} v_{q}(k-1)+\left(1-\delta_{q}\right)\left(A_{q} y(k-1)+B_{q} e(k-1)\right), \text { for } q=1,2, \ldots, 22
\end{array}\right.
$$

where, for a sampling period $T=0.002 \mathrm{~s} \delta_{q}=\exp \left(-T p_{q}\right)=\exp \left(-10^{(0.5529 q-9.4146)}\right)$ (for $1 \leqslant q \leqslant 22$ ). Fig. 9 shows the step responses of the linear SISO fractional order system described by the linear fractional order differential equation of equation (63) 
using the MATLAB function fode_sol () [4] and using the proposed approximation structure of equation (68).

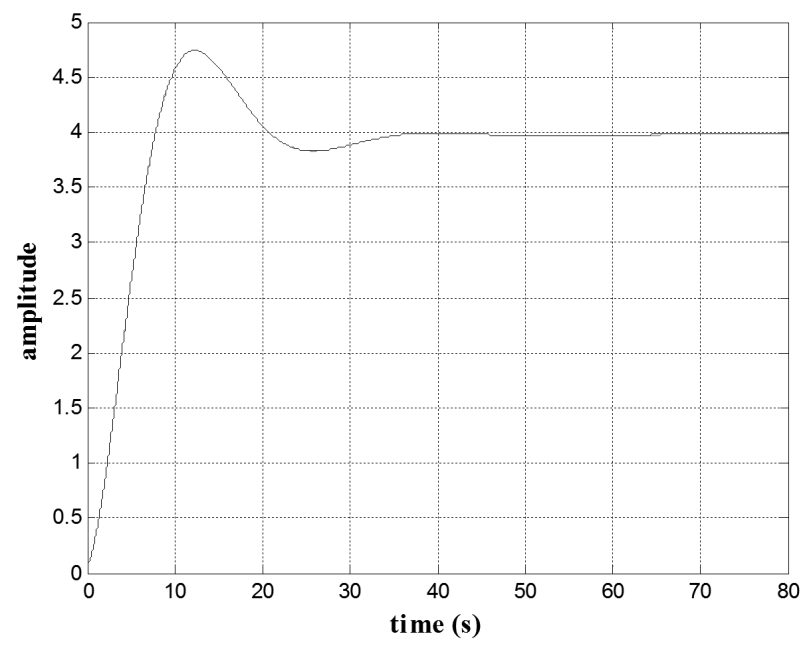

Figure 9: Step responses of the fractional order system of eqn. (63) using the proposed structure and the MATLAB function fode_sol ()

- using MATLAB function fode_sol () [24]:

for $e(t)=u(t)$, the step response of the linear fractional order differential equation of equation (63) is obtained as:

$a=\left[\begin{array}{llll}1 & 20 & 3 & 2\end{array}\right] ; \quad n a=[\operatorname{sqrt}(5) \quad \operatorname{squt}(3) \quad 0.850] ; \quad b=\left[\begin{array}{ll}3 & 8\end{array}\right] ;$ $\mathrm{nb}=[1.360] ; \mathrm{t}=0: 0.002: 80 ; \mathrm{u}=$ ones $(\operatorname{size}(\mathrm{t}))$

$y=$ fode_sol $(a, n a, b, n b, u, t)$

The digital simulation of the linear SISO fractional order system described by the linear fractional order differential equation of equation (63) is given by:

$$
\left\{\begin{array}{l}
\left\{\begin{array}{l}
v_{q}(k)=\left(1-\delta_{q}\right) x_{q}(k-1) \\
x_{q}(k)=-\delta_{q} x_{q}(k-1)+\left(A_{q} y(k)+B_{q} e(k)\right)
\end{array}, \text { for } q=1,2, \ldots, 22\right. \\
y(k)=\sum_{q=1}^{N} v_{q}(k)
\end{array}\right.
$$

where, for $1 \leqslant q \leqslant 22, \delta_{q}, A_{q}$ and $B_{q}$ are as above; and the sampling period $T=0.002 \mathrm{~s}$.

In the second example a more oscillatory linear SISO fractional order system 
is considered. It is described by the following linear fractional order differential equation:

$$
D^{2.45} y(t)+10 D^{1.87} y(t)+D^{0.58} y(t)+10 y(t)=10 e(t)
$$

whose transfer function is given as:

$$
H(s)=\frac{Y(s)}{E(s)}=\frac{10}{s^{2.45}+10 s^{1.87}+s^{0.58}+10} .
$$

For $N=22$ and for $\left[\omega_{L}, \omega_{H}\right]=[0.01 \mathrm{rad} / \mathrm{s} 1000 \mathrm{rad} / \mathrm{s}]$ the parameters $\omega_{c}, \omega_{\max }$ and $\varepsilon$ are:

$$
\omega_{c}=0.005 \omega_{L}=5 \cdot 10^{-5}, \quad \omega_{\max }=100 \omega_{H}=10^{6}, \quad \varepsilon=0.0579
$$

Then, for a sampling period $T=0.002 \mathrm{~s}$, the numerical solution of the linear fractional order differential equation of equation (70) is given as follows:

$$
\left\{\begin{array}{l}
v_{q}(k)=-\delta_{q} v_{q}(k-1)+\left(1-\delta_{q}\right)\left(A_{q} y(k-1)+B_{q} e(k-1)\right), \text { for } q=1,2, \ldots, 22 \\
y(k)=\sum_{q=1}^{N} v_{q}(k)
\end{array}\right.
$$

where, for $1 \leqslant q \leqslant 22, \delta_{q}, A_{q}$ and $B_{q}$ are given as:

$\delta_{q}=\exp \left(-T p_{q}\right), A_{q}=-\left\{h_{q}(0.58)+h_{q}(1.87)+10 h_{q}(2.45)\right\}, B_{q}=10 h_{q}(2.45)$ with

$$
\begin{gathered}
p_{q}=10^{(0.4630 q-4.6482)} \\
h_{q}(0.58)=\left(3.0472 \cdot 10^{2}\right) \frac{\prod_{p=1}^{21}\left(1-10^{0.4630(q-p-0.58)}\right)}{\prod_{\substack{p=1 \\
p \neq q}}^{22}\left(1-10^{0.4630(q-p)}\right)} \\
h_{q}(1.87)=\left(2.8177 \cdot 10^{7}\right) \frac{\prod_{p=1}^{21}\left(1-10^{0.4630(q-p-1.87)}\right)}{\prod_{\substack{22 \\
p=1}}\left(1-10^{0.4630(q-p)}\right)} \\
h_{q}(2.45)=\left(2.7008 \cdot 10^{9}\right) \frac{\prod_{\substack{21 \\
p=1}}^{22}\left(1-10^{0.4630(q-p-2.45)}\right)}{\prod_{p=1}^{22}\left(1-10^{0.4630(q-p)}\right)} \\
p \neq q
\end{gathered}
$$




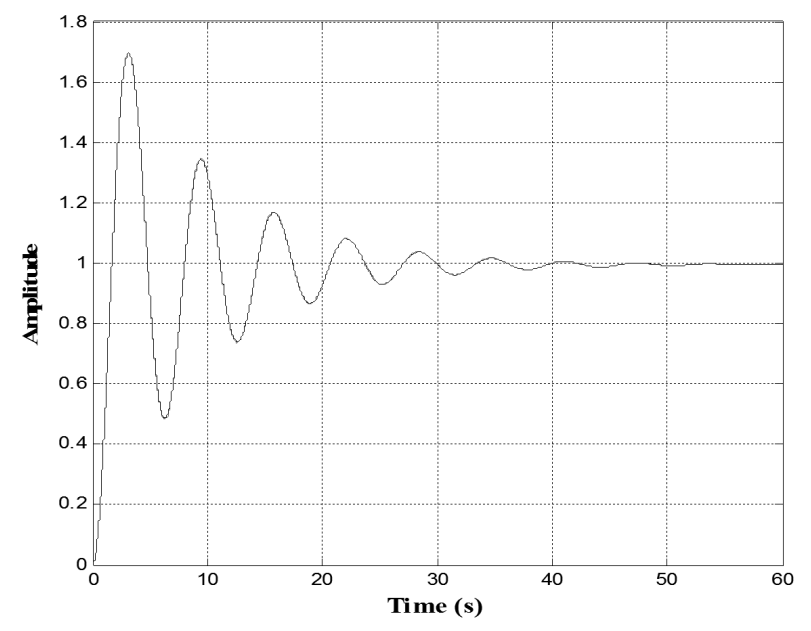

Figure 10: Step responses of the fractional order system of eqn. (70) using the proposed structure and the MATLAB function fode_sol ( )

Fig. 10 shows the step responses of the linear SISO fractional order system of equation (70) using the MATLAB function fode_sol ( ) [24] and using the proposed approximation structure of equation (72).

- using MATLAB function fode_sol ( ) [24]:

for $e(t)=u(t)$, the step response of the linear fractional order differential equation of equation (70) is obtained as:

$\mathrm{a}=\left[\begin{array}{llll}1 & 10 & 1 & 10\end{array}\right] ; \quad \mathrm{na}=\left[\begin{array}{llll}2.45 & 1.87 & 0.58 & 0\end{array}\right] ; \mathrm{b}=\left[\begin{array}{ll}1 & 0\end{array}\right] ; \mathrm{nb}=[0] ;$ $t=0: 0.002: 60 ; u=$ ones (size $(t))$

$y=f_{0 d e \_s o l}(a, n a, b, n b, u, t)$

\section{Conclusion}

In this paper, an original structure of the fractional order integrator has been presented to facilitate the analog and the digital simulations of the fractional order integrators and systems as well as the numerical resolution of the linear fractional order differential equations. The proposed simulation structure of the fractional integrator $s^{-m}$ is composed of two parts. The right part is a fixed structure made of parallel first order regular systems which are completely independent of the fractional order $m$ designed only once for $m=0.5$. So, this part can be used for the simulation of the fractional integrator of any fractional order $m>0$. The left part is a structure composed of an ensemble 
of functions depending on the fractional order $m$ only. Then, the proposed fractional order integrator structure has been extended to the analog and digital simulations as well as the resolution of the linear fractional order systems described by the linear fractional order differential equation $\sum_{i=0}^{L} a_{i} D^{\alpha_{i}} y(t)=\sum_{j=0}^{M} b_{j} D^{\beta_{j}} e(t)$. The analog or digital simulation structures obtained are also composed of two connected parts. The right part is exactly the right part of the fractional order integrator. In this case it is also completely independent of the fractional orders $\alpha_{i}(0 \leqslant i \leqslant L)$ and $\beta_{i}(0 \leqslant j \leqslant M)$ of the above differential equation; so it can be used for the simulation of any linear SISO fractional order system described by the linear fractional order differential equation. The left part is an ensemble of functions depending on the derivative orders $\alpha_{i}(0 \leqslant i \leqslant L)$ and $\beta_{i}(0 \leqslant j \leqslant M)$ and the model coefficients $a_{i}$ (for $\left.1 \leqslant i \leqslant L\right)$ and $b_{i}(0 \leqslant j \leqslant M)$ of the above differential equation.

Some illustrative examples have been presented to show the efficiency and the effectiveness of the proposed simulations and resolution techniques. The step responses of the fractional integrator and the fractional order system using the proposed method are compared to those obtained using the Grunwald-Letnikov's fractional derivative definition. The comparison results were very satisfactory.

It is also worth mentioning that the proposed structures have practical significance to circuit designers who would be interested in the hardware implementation of the linear fractional order operators and systems in the fields of control system, signal processing and identification. In the future, the use of the proposed fractional order integrator structure for the simulation of variable order integrators and systems will be investigated.

\section{References}

[1] M. Aoun, R. Malti, F. Levron and A. Oustaloup: Numerical simulations of fractional systems: An overview of existing methods and improvements. Nonlinear Dynamics, 38 (2004), 117-131.

[2] D. Baleanu, J.A.T Machado and A.C.J. Luo (Eds.): Fractional Dynamics and Control. Springer-Verlag, New York, 2012.

[3] D. Boucherma, A. Charef, H. Nezzari: The solution of state space linear fractional system of commensurate order with complex eigenvalues using regular exponential and trigonometric functions. Int. J. of Dynamics and Control, 5(1), (2017), 79-94.

[4] A. CHAREF: Modeling and analog realization of the fundamental linear fractional order differential equation. Nonlinear Dynamics, 46 (2006), 195-210.

[5] A. Charef and H. NezZari: On the fundamental linear fractional order differential Eequation. Nonlinear Dynamics, 65 (2011), 335-348. 
[6] A. CHAREF: Analogue realization of fractional order integrator, differentiator and fractional $\mathrm{PI}^{\lambda} \mathrm{D}^{\mu}$ controller. IEE Proc. on Control Theory and Applications, 153 (2006), 714-720.

[7] A. CHAREF and D. IDIOU: Design of analog variable fractional order differentiator and integrator. Nonlinear Dynamics, 69 (2012), 1577-1588.

[8] A. Charef, H.H. Sun, Y.Y. Tsao and B. Onaral: Fractal system as represented by singularity function. IEEE Trans. on Automatic Control, 37 (1992), 1465-1470.

[9] Y.Q. Chen and B.M. VINAGRE: A new IIR-type digital fractional order differentiator. Signal Processing, 83 (2003), 2359-2365.

[10] M. DAliR and M. BASHOuR: Applications of fractional calculus. Applied Mathematical Sciences, 4 (2010), 1021-1032.

[11] S.K. DAmarla and M. Kundu: Numerical solution of multi order fractional differential equations using generalized triangular function operational matrices. Applied Mathematics and Computation, 263 (2015), 189-203.

[12] S. DAS and I. PAN (EDS.): Fractional Order Signal Processing: Introductory Concepts and Applications. Springer, New York, 2012.

[13] A. Djouambi, A. Charef and T. BouktiR: Fractional order robust control and $\mathrm{PI}^{\lambda} \mathrm{D}^{\mu}$ controllers. WSEAS Trans. on Circuits and Systems, 8 (2005), 850-857.

[14] R. GARRA and F. Polito: Analytic solutions of fractional differential equations by operational methods. Applied Mathematics and Computation, 218 (2012), 10642-10646.

[15] K. Hamdaoui and A. Charef: A new discretization method for fractional order differentiators via bilinear transformation. 15th Int. Conf. on Digital Signal Processing, (2007), 280-283.

[16] Y. HU, Y. LUO and Z. LU: Analytical solution of the linear fractional differential equation by Adomian decomposition method. J. of Computational and Applied Mathematics, 215 (2008), 220-229.

[17] C.X. Jiang, J.E. Carletta, T.T Hartley and R.J. Veillette: A systematic approach for implementing fractional-order operators and systems. IEEE J. on Emerging and Selected Topics in Circuits and Systems, 3 (2013), 301-312.

[18] T. KACZOREK: Realization problem for fractional continuous time systems. Archives of Control Sciences, 18(1), (2008), 43-58. 
[19] M.M. KHADER: A new fractional Chebyshev FDM: an application for solving the fractional differential equations generated by optimization problem. Int. J. of Systems Science, 46 (2015), 2598-2606.

[20] S. Kumar, K. Singh and R. SAxena: Closed-form analytical expression of fractional order differentiation in fractional Fourier transform domain. Circuits, Systems, and Signal Processing, 32 (2012), 1875-1889.

[21] C. LI, Y. LI, A. CHEN and J. YE: Numerical approaches to fractional calculus and fractional ordinary differential equation. J. of Computational Physics, 230 (2011), 3352-3368.

[22] X. LI: Numerical solution of fractional differential equations using cubic B-spline wavelet collocation method. Communications in Nonlinear Science and Numerical Simulation, 17 (2012), 3934-3946.

[23] R.L. MAgin: Fractional calculus in bioengineering. Begell House, Redding, 2006.

[24] A. Monje, Y.Q. Chen, B.M. Vinagre, D. Xue and V. Fellin: Fractionalorder Systems and Controls Fundamentals and Applications. Springer, London, 2010 .

[25] Z.M. ОвIDAT: Analytic study on linear systems of fractional differential equations. Computers and Mathematics with Applications, 59 (2010), 1171-1183.

[26] K. Ogata: Discrete-time control systems. Prentice Hall. Englewood Cliffs, 1987.

[27] K. OPRZEDKIEWICZ: Approximation method for a fractional order transfer function with zero and pole. Archives of Control Sciences, 24(4), (2014), 447-463.

[28] G. OtURAnC, A. KURNAZ and Y. Keskin: A new analytical approximate method for the solution of fractional differential equations. Int. J. of Computer Mathematics, 85 (2008), 131-142.

[29] A. Oustaloup: La Commande CRONE, Editions Hermés, Paris, 1991.

[30] F. PAdula and A. VAsioli: Advances in Robust Fractional Control. Springer, New York, 2015.

[31] I. Petras: Fractional-Order Nonlinear Systems: Modeling, Analysis and Simulation. Springer-Verlag, Berlin, 2011.

[32] A. SaAdatmandia and M. Dehghanb: A new operational matrix for solving fractional order differential equations. Computers and Mathematics with Applications, 59 (2010), 1326-1336. 
[33] J. Sabatier, O.P. Agrawal and J.A. Tenreiro Machado(Eds.): Advances in Fractional Calculus: Theoretical Development and Applications in Physics and Engineering. Springer, Dordrecht, 2007.

[34] H. Sheng, Y.Q. Chen and T.S QIU: Fractional Processes and Fractional-Order Signal Processing: Techniques and Applications. Springer-Verlag, Berlin, 2012.

[35] M.P. Tripathi, V.K. Baranwal, R.K. Pandey and O.P. Singh: A new numerical algorithm to solve fractional differential equations based on operational matrix of generalized hat functions. Communications in Nonlinear Science and Numerical Simulation, 18 (2013), 1327-1340.

[36] C.C. TsEnG: Design of FIR and IIR fractional order Simpson digital integrators. Signal Processing, 87 (2007), 1045-1057. 